\title{
Glutathione peroxidase 3 (GPX3) expression predicts the prognosis of numerous malignant tumors: A pan- cancer Analysis
}

\section{Yuetong Wang}

Shaanxi Academy of Traditional Chinese Medicine

\section{Guotao Fu}

Shaanxi University of Chinese Medicine

\section{Xueqin Chen}

Shaanxi University of Chinese Medicine

\section{Zengrun Xia}

Ankang R\&D Center of Se-enriched Products

\section{Meng Qi}

Ankang R\&D Center of Se-enriched Products

Xiaoping Du

Ankang R\&D Center of Se-enriched Products

\section{Kun Liu}

Binzhou Medical University

Qiling Liu

Shaanxi University of Chinese Medicine

\section{$\mathrm{Na}$ Sun}

Shaanxi University of Chinese Medicine

\section{Chuandao Shi}

Shaanxi University of Chinese Medicine

Rongqiang Zhang ( $\sim$ Zhangrqxianyang@163.com )

Shaanxi University of Chinese Medicine

Kai Qu

Shaanxi Provincial Hospital of Chinese Medicine

\section{Research}

Keywords: Gene expression, Glutathione peroxidase 3 (GPX3), Tumors, Biomarkers

Posted Date: October 15th, 2021

DOl: https://doi.org/10.21203/rs.3.rs-845660/v2

License: (9) This work is licensed under a Creative Commons Attribution 4.0 International License. Read Full License 


\section{Abstract \\ Background}

Malignant tumor is a general term for uncontrollable growth of cells. Several studies have investigated that role of GPX3 in tumors. However, no pan-cancer analysis has be conducted to assess the diagnostic and prognostic potential of GPX3.

\section{Methods}

GPX3 mRNA and protein expression profile was analyzed in the TCGA + GTEx database and CPTAC database. Kaplan-Meier survival curves and forest plots were constructed to help evaluate the impact of GPX3 on the survival and prognosis of cancer patients. Gene mutations of GPX3 were analyzed based on TMB/MSI. The correlation between GPX3 and tumor immune infiltration was assessed using TIMER2. Enrichment analysis was performed to determine tumor-related signaling pathways associated with GPX3. A prediction model for STAD was established.

\section{Results}

GPX3 was downregulated in most malignant tumors, and was significantly associated with the survival and prognosis of malignant tumors, such as STAD, PAAD, COAD, etc. Moreover, GPX3 expression was positively correlated and negatively with MSI in 13 and 20 categories of tumor respectively after GPX3 expression was positively correlated and negatively with TMB in 9 and 24 tumors separately $(P<0.05)$. A positive correlation was found between GPX3 and the infiltration level of major immune cells and Cancer-associated fibroblasts $(P<0.05)$. The effects of GPX3 were mediated by the AMPK signaling pathway, fructose and mannose metabolism.

\section{Conclusions}

This is a novel pan-cancer analysis on the relationship between GPX3 and human tumors. Findings of this research will deepen our understanding on the role of GPX3 in the development, regulation and prognosis of malignant tumors.

\section{Background}

Malignant tumor is a general term for uncontrollable growth of cells. Here, cells do not respond to normal regulatory signals, grows and differentiates abnormally and display local tissue invasion and distant metastasis [1]. There are currently about 260 types of tumors in humans. According to the latest global cancer statistics by the World Health Organization, in 2020 alone, there were 19.29 million new cancer cases worldwide, resulting in 9.96 million deaths ${ }^{[2]}$. Cancers present a huge economic burden to patients, their families and even the nation, threatening both social and economic development. Cancer is highly fatal and seriously threatens human health and life. Even with the recent advances in diagnosis and treatment, the occurrence and mortality due to of malignant tumors are still increasing. Biomarkers are potentially accurate and effective diagnostic and therapeutic targets for malignant tumors. 
Glutathione peroxidase (GPX) is an important protein that scavenges reactive oxygen species (ROS) in organisms [3]. Glutathione peroxidase 3 (GPX3) is the only known extracellular glycosylase in the glutathione peroxidase family and contains the selenocysteine residues. It defends against cellular stress signals and reactive oxygen species, thereby maintaining the cellular genetic integrity ${ }^{[4]}$. Moderate GPX3 expression is necessary to maintain normal metabolism and physiology and induction of important pathological changes in certain organs. However, abnormal expression of GPX3 results in the occurrence and development of various malignant tumors in the body. For instance, recent researches have demonstrated the abnormal expression of GPX3 in esophageal cancer

${ }^{[5]}$, melanoma ${ }^{[6]}$, colon cancer ${ }^{[7]}$, gastric cancer ${ }^{[8]}$, ovarian cancer ${ }^{[9]}$ and other malignant tumors. Even so, studies on the role of GPX3 are relatively few and have only assessed the role of GPX3 in a small number of malignant tumors.

Pan-Cancer Analysis Project is a collaborative initiative that integrates, analyzes and interprets The Cancer Genome Atlas (TCGA) data of different malignant tumors from different platforms ${ }^{[10,11]}$. Pan-cancer analyses can not only reveal common phenotypic characteristics of malignant tumors, but can also unravel molecular events underlying the development of tumors and corresponding internal regulatory mechanisms. These studies are also important in unraveling the complex tumorigenesis as well as possible molecular and target genes relevant to clinical prognosis of cancers. However, even with the large amount of clinical data, there is no pancancer evidence on the relationship between GPX3 and several tumor types.

Herein, we first analyzed the expression of GPX3 in 33 different malignant tumors using RNA-seq data. Then we analyzed the correlation between GPX3 expression and tumor stage. The effect of GPX3 on the survival and prognosis of malignant tumor was also investigated. We explored the relationship between GPX3 expression and infiltration of immune cells. we also performed bioinformatics analyses to explore potential mechanism underlying GPX3 expression and development of human malignant tumors. Finally, we verified the expression pattern and prognostic significance of GPX3 in STAD and COAD, and proposed a prediction model for STAD. Findings of this research will deepen our understanding on the role of GPX3 in the development, regulation and prognosis of malignant tumors. It can also uncover potential biomarkers for early diagnosis, prevention and treatment of malignant tumors.

\section{Materials And Methods}

\subsection{Expression of GPX3 in normal human tissues}

The Human Protein Atlas (HPA) (https://www.proteinatlas.org/) is a comprehensive repository for protein expression profiles in tissue, cells and blood and their metabolic and pathologic roles in the body. The expression of GPX3 in normal tissues was analyzed using HPA RNA-seq tissue data ${ }^{[12]}$. The expression of GPX3 protein in main cancer tissues (Colorectal cancer, Prostate cancer, Breast cancer, Lung cancer, Liver cancer) and normal tissues (Normal kidney tissues) was analyzed using immunohistochemical (IHC) tissue images in HPA.

\subsection{Gene expression analysis}

The expression of GPX3 mRNA in 33 different malignant tumors and corresponding normal tissues was assessed using RNA sequence data in the TCGA and GTEx databases ${ }^{[13]}$. Differential gene expression between cancerous and corresponding normal tissues was analyzed using t-tests, whereas the differently expressed genes between 
the tissue sets were presented using a violin plot. Before plotting, the expression data was first transformed to $\log 2$ [TPM (Transcripts per million) + 1], with $P<0.05$ considered statistically significant.

The cancer omics data from the Clinical proteomic tumor analysis consortium (CPTAC) was analyzed using UALCAN platform (http://ualcan.path.uab.edu/analysis-prot.html) ${ }^{[14]}$. The major analysis involved the expression of GPX3 several malignant tumors including Breast cancer, Ovarian cancer, Colon cancer, Clear cell Renal Cell Carcinoma (Clear cell RCC), Uterine Corpus Endometrial Carcinoma (UCEC) and Lung adenocarcinoma (LUAD). The degree and nature of abnormal expression of GPX3 protein between cancer and adjacent normal tissues was based on Z-values, with the median protein expression levels used as reference points.

The GPX3 expression at different cancer stages was analyzed using the "Expression DIY" module in GEPIA2 platform (http://gepia2.cancer-pku.cn/\#index). The corresponding violin plot was also constructed after transformation of the expression data to $\log _{2}(T P M+1)$. The comparative analyses for the expression of GPX3 in different cancer stages were performed to understand the role of the protein in cancer pathology [15].

\subsection{Prognostic utility of GPX3}

We constructed the predictive potential of GPX3 for Overall Survival (OS), Disease Specific Survival (DSS), Disease-Free Interval (DFI) and Progression-Free Interval (PFI) of different tumors in the TCGA database. The median GPX3 expression was used as the cutoff level for high and low expression of the protein. The predictive utility of GPX3 for OS, DSS, DFI and PFI of cancer patients was assessed using log-rank test and the Kaplan-Meier curve.

Further analyses were performed to assess epidemiological implication of GPX3 expression in 33 tumor types in the TCGA database. The effect of GPX3 expression on Overall Survival (OS), Disease-Free Survival (DFS), Progression Free Survival (PFS) and Disease Specific Survival (DSS) for different cancers were assessed using $\mathrm{R}$ software V. 4.0.3. The relationship between GPX3 expression and OS, DFS, PFS and DSS were analyzed using univariate Cox regression analysis and hazard ratios (HR) at 95\% confidence interval (Cl) and log-rank $P$ test at statistical significance of $P<0.05^{[16]}$.

\subsection{Genetic alteration in tumor cells}

RNA-seq data for 33 cancer patients in TCGA database were downloaded from the genomic data Commons (GDC) portal (https://portal.gdc.cancer.gov/). Tumor Mutation Burden (TMB), defined as the number of mutations (insertion/deletion) per mega base in the exon coding region of a gene, was analyzed as previously described ${ }^{[17]}$. The TMB is directly proportional to the expression of neoantigens recognizable by $T$ cells, which influences the immune response. Microsatellite Instability (MSI) is any change in the microsatellite length caused by insertion or deletion of a repeat unit in a gene in a tumor tissue, relative to normal tissue ${ }^{[18]}$, which generates microsatellite alleles. TMB and MSI are often used in assessing the prognosis and effect of immunotherapies. The association between GPX3 expression and TMB and MSI in cancerous tissues was assessed. Relevant data was obtained from the TCGA database; whereas the analysis was performed using R software V. 4.0.3, with statistical significance sets at $P<0.05$.

\subsection{Infiltration of immune cells}

Tumor Immune Evaluation Resource 2 (TIMER2) is a database for the systematic analysis of immune infiltration of different cancer types (B cells, CD4 + T cells, CD8 + T cells, Neutrophils, Macrophages, and Dendritic cells). In 
this study, infiltrating immune cell scores of 33 cancers were downloaded from the TIMER2 database. Spearman correlation analysis was used to evaluate the correlation between GPX3 expression and scores of B cells, CD4 + T cells, CD8 + T cells, Neutrophils, Macrophages, and Dendritic cells ${ }^{[19]}$.

The relationship between GPX3 expression and infiltration levels of Cancer associated fibroblasts (CAFs) was analyzed using TIMER2 platform ( http://timer.cistrome.org/ ). CAF regulates functioning of immune cells in the tumor microenvironment (TME). The infiltration of immune cells in the TME were estimated using EPIC, MCPCOUNTER, XCELL and TIDE algorithms. Since most immune cell types are negatively correlated with tumor purity, we obtained $P$-values and correlation coefficient by Spearman's rank correlation test after purity adjustment. The above relationship was presented using a heat map and a scatter plot. Scatter plot was constructed for cells exhibiting the strongest correlation with tumor $(P<0.05) .{ }^{[15]}$

\subsection{Enrichment analysis}

The top 100 genes associated with GPX3 expression in TCGA and GTEx databases were identified based on Pearson's correlation coefficient (PCC). The correlations between GPX3 and the top 3 most dysregulated genes were also assessed using GEPIA2 module. A scatter plot for the top 3 most dysregulated genes was also constructed.

Protein-protein interaction (PPI) network in tumor tissues associated with GPX3 expression was constructed using STRING platform (https://string-db.org/). The minimum required interaction score was set as Low confidence = 0.150 , the max number of interactors to show was set as no more than 50 interactors in 1 st shell. Finally, the available experimentally determined GPX3-binding proteins were obtained.

KEGG (Kyoto Encyclopedia of Genes and Genomes) pathway enrichment analysis of the 150 genes, which was combined from top 100 GPX3-similar genes and 50 GPX3-interacted genes, was performed to identify pathways regulated by the proteins. The resultant genes were uploaded to DAVID database, under the name of ("OFFICIAL_GENE_SYMBOL") and ("Homo sapiens") for species. GO (Gene Ontology) enrichment analysis for the Biological Process (BP), Cellular Component (CC) and Molecular Function (MF) associated with the dysregulated genes were also identified and plotted graphically using the cnetplot package (circular $=\mathrm{F}$, colorEdge $=\mathrm{T}$, node_label = T). KEGG and GO analyses were performed using R software. Statistical significance for both analyses was set at two-tailed $P<0.05^{[15]}$.

\subsection{Construction and validation of the nomogram of GPX3 for STAD}

The results above indicated that GPX3 expression had an important impact on the survival prognosis of numerous malignant tumors, such as BLCA, COAD, PAAD, STAD. OS, DSS, PFS, DFS, DFI and PFI all strongly supported that the prognosis of STAD would get worse when GPX3 level elevated. Therefore, a nomogram of GPX3 for STAD was established and verified to further analyze the predictive significance of GPX3 for the OS of patients with STAD. Firstly, univariate and multivariate Cox regression analysis were used to identify all independent factors for STAD and displayed as hazard ratios (HR) combined with the corresponding 95\% confidence intervals $(\mathrm{Cl})$. Then, according to the results of the multivariate Cox regression analysis model, a prognostic nomogram was established to predict the OS probability of STAD patients at 1-, 2-, 3-, and 5-year by the TCGA training dataset by using the rms package in R software. Concordance index (C-index), which ranges from 0.5 (poor) to 1.0 (perfect), was employed to assess the performance of nomogram. Briefly, the higher the C- 
index, the better its prognostic accuracy. Finally, to ensure the nomograms' accuracy, calibration and validation of the nomogram were performed using the R package "rms" and "cmprsk", $P<0.05$ was considered statistically significant ${ }^{[20,21]}$.

\section{Results}

\subsection{Expression of GPX3 in normal human tissues}

GPX3 gene is strongly expressed in Kidney, Thyroid gland and Adipose tissues, etc. (Fig. 1A). GPX3 protein was detected in carcinoid, colorectal cancer, prostate cancer, renal cancer, skin cancer and lymphomas (Fig. 1B). The IHC images for GPX3 protein in colorectal cancer, prostate cancer, breast cancer, lung cancer, liver cancer and normal kidney tissues are shown in Fig. $1 \mathrm{C}-\mathrm{H}$. Detailed clinical information of the tissue donors for $\mathrm{IHC}$ analyses are summarized in Table 1.

Table 1

Key clinical information of subjects for immunohistochemical experiment

\begin{tabular}{|c|c|c|c|c|c|c|c|}
\hline Protein & Tissue & Histological type & Age & Gender & Location & Quantity & Intensity \\
\hline GPX3 & Colorectal & Adenocarcinoma & 78 & female & $\begin{array}{l}\text { Cytoplasmic/ } \\
\text { membranous } \\
\text { nuclear }\end{array}$ & $>75 \%$ & Moderate \\
\hline GPX3 & Breast & Duct carcinoma & 61 & female & $\begin{array}{l}\text { Cytoplasmic/ } \\
\text { membranous }\end{array}$ & $>75 \%$ & Weak \\
\hline GPX3 & Prostate & Adenocarcinoma & 68 & male & $\begin{array}{l}\text { Cytoplasmic/ } \\
\text { membranous }\end{array}$ & $<25 \%$ & Strong \\
\hline GPX3 & Lung & $\begin{array}{l}\text { Squamous cell } \\
\text { carcinoma }\end{array}$ & 64 & male & Nuclear & $<25 \%$ & Weak \\
\hline GPX3 & Liver & $\begin{array}{l}\text { Carcinoma/ } \\
\text { Hepatocellular }\end{array}$ & 73 & female & $\begin{array}{l}\text { Cytoplasmic/ } \\
\text { membranous }\end{array}$ & $<25 \%$ & Moderate \\
\hline \multirow[t]{2}{*}{ GPX3 } & glomeruli & Normal tissues & 61 & male & Cytoplasmic/ & $<25 \%$ & Weak \\
\hline & tubules & & & & $\begin{array}{l}\text { Membranous } \\
\text { nuclear }\end{array}$ & $>75 \%$ & Strong \\
\hline
\end{tabular}

\subsection{GPX3 gene expression in different tumors}

Analysis of data in TCGA and GTEx databases revealed that GPX3 gene was under expressed in 22 of 34 tumor types including Adrenocortical carcinoma (ACC), Bladder Urothelial Carcinoma (BLCA), Breast invasive carcinoma (BRCA), Cervical squamous cell carcinoma and endocervical adenocarcinoma (CESC), Cholangiocarcinoma (CHOL), Colon adenocarcinoma (COAD), Esophageal carcinoma (ESCA), Head and Neck squamous cell carcinoma (HNSC), Kidney Chromophobe (KICH), Kidney renal clear cell carcinoma (KIRC), Kidney renal papillary cell carcinoma (KIRP), Lung adenocarcinoma (LUAD), Lung squamous cell carcinoma (LUSC), Ovarian serous cystadenocarcinoma (OV), Prostate adenocarcinoma (PRAD), Rectum adenocarcinoma (READ), Skin Cutaneous 
Melanoma (SKCM), Stomach adenocarcinoma (STAD), Testicular Germ Cell Tumors (TGCT), Thyroid carcinoma (THCA), Uterine Corpus Endometrial Carcinoma (UCEC) and Uterine Carcinosarcoma (UCS). Among them, analyses were performed at statistical significance of $P<0.01$ for $\mathrm{CHOL}$ and $P<0.001$ for the rest of the tumors. Expression of GPX3 gene was relatively higher Lymphoid Neoplasm Diffuse Large B-cell Lymphoma (DLBC), Glioblastoma multiforme (GBM), Glioma (LGG), Liver hepatocellular carcinoma (LIHC) (all at $P<0.001)($ Fig. 2).

As shown in Fig. 3A, analysis of CPTAC data revealed that compared with normal tissues, GPX3 was under expressed in Breast cancer, Ovarian cancer, Colon cancer, Clear cell RCC, UCEC, and Lung adenocarcinoma $(P<$ 0.001).

Further analyses revealed that GPX3 expression levels correlated with pathological stages of eight cancers including ACC, BLCA, KIRC, KIRP, LIHC, Pancreatic adenocarcinoma (PAAD), READ and THCA (all at $P<0.05$ ) (Fig. 3B). The expression of GPX3 was highest in stage I and lowest in stage II of ACC. In BLCA, GPX3 expression was highest in stage III, lowest in stage II and moderate in stage IV. There were no significance differences in GPX3 expression between stage I and III of KIRC, the same as stage II and IV. In KIRP, GPX3 expression increased gradually between stage I-III but decreased slightly in stage IV. In LIHC, GPX3 expression was highest in stage IV and lowest in stage III, In PAAD, GPX3 expression was highest in stage III and lowest in stage II whereas in READ, GPX3 expression increased from stage I to stage IV. In THCA, GPX3 expression was highest in stage II but lowest in stage IV.

\subsection{Prognostic value of GPX3}

Cancer patients were divided into high and low GPX3 expression groups. The GPX3 expression predicted the OS of four tumor types. Among them, high GPX3 expression conferred longer OS of patients with PAAD $(P=0.0031)$, whereas low GPX3 expression was linked to longer OS of patients with BLCA $(P=0.0049), \operatorname{COAD}(P=0.0078)$ and STAD $(P=0.00041)$ (Fig. 4A). The GPX3 expression also predicted the DSS of four tumor types and high GPX3 expression conferred longer DSS of patients with PAAD $(P=0.0091)$, whereas low GPX3 expression was linked to longer DSS of patients with $\operatorname{BLCA}(P=0.1), \operatorname{COAD}(P=0.00026)$ and STAD $(P=0.00028)$ (Fig. 4B). The GPX3 expression predicted the DFI of three tumor types. Low GPX3 expression was linked to longer DFI of patients with BRCA $(P=0.012)$ LUAD $(P=0.004)$ and STAD $(P=0.00016)$ (Fig. 4C). Meanwhile, high-expression of GPX3 was associated with longer PFI of PAAD $(P=0.0037)$. Contrarily, low expression of GPX3 was associated with longer PFI of patients with COAD $(P=0.0036)$ and STAD $(P<0.0001)$ cancers (Fig. 4D).

Univariate survival analysis revealed that high-expression of GPX3 was related to poor prognosis of STAD (HR = 1.002, 95\%Cl: 1-1.003, $P=0.0125)$ but better prognosis of PAAD (HR=0.998, 95\%Cl: 0.997-1, $P=0.0134)(\mathrm{Fig} .5 \mathrm{~A})$. Meanwhile, over GPX3 expression was linked with DFS of COAD (HR=1.003, 95\%Cl: $1.001-1.006, P=0.0105)$, STAD (HR = 1.002, 95\%Cl: 1.001-1.003, $P=0.0048)$ and PAAD (HR=0.998, 95\%Cl: 0.997-1, $P=0.0067)($ Fig. 5B). Moreover, over-expression of GPX3 was associated with PFS of patients with COAD (HR $=1.004,95 \% \mathrm{Cl}$ : 1.0011.007, $P=0.0028)$, STAD (HR = 1.002, 95\%Cl: 1-1.003, $P=0.0151)$ and PAAD (HR= 0.998, 95\%Cl: 0.997-1, $P=$ $0.0134)$ (Fig. 5C). Also, high-expression of GPX3 was associated with poor prognosis of $\mathrm{BRCA}(\mathrm{HR}=1.002,95 \% \mathrm{Cl}$ : 1.001-1.004, $P=0.0068)$ and STAD (HR $=1.003,95 \% \mathrm{Cl}: 1.001-1.006, P=0.0069)$ but better prognosis of LUAD $(\mathrm{HR}=0.998,95 \% \mathrm{Cl}: 0.997-1, P=0.0322)($ Fig. 5D).

Overall, these findings demonstrate that GPX3 expression levels influence the prognosis of several tumors, particular STAD, and COAD PAAD. Therefore, we focused on these 3 types of tumors in subsequent analyses, 
especially on STAD, and COAD due to larger sample sizes.

\subsection{The correlation between GPX3 expression and TMB/MSI}

GPX3 expression positively correlated with TMB of nine tumor types including THYM, Acute Myeloid Leukemia (LAML), LIHC, KIRC, KIRP, COAD, TGCT, SARC and PCPG. The strongest correlation was observed for THYM, LAML and LIHC. Contrarily, GPX3 expression negatively correlated with TMB of 24 tumor types including PRAD, PAAD, STAD, SKCM, UCS, ESCA, LUAD, KICH, GBM, LUSC, Uveal Melanoma (UVM), THCA, Mesothelioma (MESO), HNSC, ACC, READ, LGG, BRCA, DLBC, BLCA, OV, CHOL, UCEC and CESC. The strongest correlation was observed for PRAD, PAAD and STAD (Fig. 6A). GPX3 expression also positively correlated with the MSI of SKCM, COAD, KIRC, BLCA, UCEC, CESC, BRCA, ESCA, THYM, THCA, LAML, READ and MESO. The strongest correlations were observed for SKCM, COAD and KIRC. However, there was a strong negatively correlation between GPX3 expression and MSI of STAD, CHOL, KICH, OV, LUSC, LUAD, GBM, UCS, Pheochromocytoma and Paraganglioma (PCPG), TGCT, PRAD, ACC, Sarcoma (SARC), HNSC, KIRP, LIHC, LGG, DLBC, PAAD and UVM. The strongest correlations were observed for STAD, CHOL and $\mathrm{KICH}$ (all Ps<0.05) (Fig. 6B).

\subsection{GPX3 expression and infiltration of Immune cells}

We investigated whether the expression of GPX3 in 33 tumors from the TIMER2 database was related to the level of immune infiltration. The results showed that the expression of GPX3 in PAAD and COAD was significantly positively correlated with B cells, CD4 + T cells, CD8 + T cells, Neutrophils, Macrophages and Dendritic cells. In STAD, the expression of GPX3 was significantly positively correlated with the infiltration levels of CD4 + T cells, CD8 + T cells, Neutrophils, Macrophages and Dendritic cells, but not with B cells (Fig. 7A).

The relationship between GPX3 expression and infiltration levels of CAFs in the 40 evaluated tumors were assessed using TIDE, XCELL, EPIC and MCPCOUNTER algorithms. We found GPX3 expression positively correlated with infiltration of CAFs in BLCA, BRCA, BRCA-Basal, BRCA-LumA, BRCA-LumB, COAD, HNSC, HNSCHPV-, PAAD, READ, SKCM-Metastasis, STAD and Thymoma (THYM). A scatter plot for the above relationships is shown in Fig. 7B. Such as, based on TIDE algorithm, GPX3 expression positively correlated with the infiltration level of CAFs in BLCA tissues (Rho $=0.394, P=4.17 \mathrm{e}-15)$.

\subsection{Key pathways linked to GPX3 expression}

Top 3 genes related to GPX3 expression based on the TCGA data were further analyzed. It was found GPX3 expression positively correlated with expression of MOCS1 $(R=0.28)$, TNS2 $(R=0.2)$ and FZD4 $(R=0.27)$ genes (Fig. 8A). Further analyses identified 24 GPX3 binding proteins. The interaction network of these proteins is shown in Fig. 8B. KEGG analyses revealed that the pathogenesis of GPX3 in tumors was related to AMPK and Fructose and mannose metabolism pathways (Fig. 8C). The GO enrichment analysis further revealed that GPX3 gene regulates Angiogenesis and vascular morphogenesis, vasculature development, tube morphogenesis among others. The gene also regulates expression of intrinsic components of plasma membrane as well as phosphatase and Phosphoric ester hydrolase activities.

\subsection{Validation analysis}

In the above prognosis analysis, GPX3 showed significant effects on STAD, COAD and PAAD, especially on STAD and COAD in OS, DFS, PFS, DFI and PFI. To verify the expression pattern and prognostic significance of GPX3 in STAD and COAD, we further retrieved two datasets from Gene Expression Omnibus (GEO)

(https://www.ncbi.nlm.nih.gov/geo/) and TCGA with the accession numbers GSE44861 and GSE29272 [22, 23]. 
The results showed that the expression level of GPX3 in STAD and COAD tumor tissues was significantly lower than that in normal tissues, which were consistent with the results of TCGA datasets. The AUCs (area under the ROC curves) for COAD was 0.6698 (95\% Cl: 0.5660-0.7736, P=0.002) from GEO dataset and 0.9909 (95\% Cl: 0.9862-0.9956, P< 0.001) from TCGA dataset (Fig. 9A). The AUCs for STAD was 0.8406 (95\%Cl: 0.7865-0.8946, $\mathrm{P}<0.001$ ) from GEO dataset and 0.9599 (95\%Cl: 0.9464-0.9734, $\mathrm{P}<0.001$ ) from TCGA dataset (Fig. 9B), respectively. The results suggested that the diagnostic values of GPX3 for STAD and COAD was the same based on GEO and TCGA database, and GPX3 performed a good diagnosis ability for the two diseases, which also demonstrated that our aforementioned results were reliable.

\subsection{Construction and verification of nomogram}

Independent prognostic factors, including PTNM stage, age, radiation therapy, and GPX3 expression were included to create prognostic nomograms for the OS of STAD patients. The nomogram showed that pTNM stage had the greatest influence on prognosis, followed by age and radiation therapy (Fig. 10A-B). In addition, the validation of the nomogram was performed by $\mathrm{C}$-index and calibration. The C-index predicted by the histogram was 0.69 (95\% Cl: 0.627-1; $P<0.001)$ (Fig. 10C). The calibration curve showed the concordant survival rate between the predicted and observed nomograms (Fig. 10D). It can be believed the prognostic nomogram established in our present study could effectively predict OS probability of patients with STAD.

\section{Discussion}

Numerous complex multi-step processes precede development of malignant tumors, induced by numerous biological, chemical and physical factors. Recent diagnosis and treatment advances have facilitated early detection and improved overall survival of cancer patients. However, prognosis modalities are still at infancy stage. Understanding molecular mechanism of tumor pathogenesis can potentially improve or provide new frontier in the diagnosis, treatment and prevention of tumors. GPX3 is a tumor suppressor gene located on chromosome 5q23. It codes for the main antioxidant enzyme in plasma which participates in detoxification of free oxygen radicals such as hydrogen peroxide, thus protecting cells from oxidative stress-induced damage. Inactivation of GPX3 leads to the accumulation of ROS, which have been found to induce oxidative deoxyribonucleic acid (DNA) damage. The resultant gene changes lead to development of cancers ${ }^{[24]}$. Lou W et al. ${ }^{25]}$ reported that GPX3 participates in the growth and metastasis of breast cancer. In a related study, Noci S et al. ${ }^{26]}$ found that $G P X 3$ expression influences the incidence, survival rate and recurrence of colorectal cancer. However, the roles of GPX3 in different tumor types are still unclear. Moreover, to the best of our knowledge, there is few pan-cancer studies on the role of GPX3 in various cancer properties. Therefore, we evaluated the role of GPX3 expression on normal human tissues, gene expression, protein expression, prognosis, gene mutation, infiltration of immune cells, associated pathways, prognostic model, etc. Data for 33 different tumor types was extracted from the TCGA, GTEx, CPTAC, HPA and other databases.

We found GPX3 expression was modulated in most tumors. In one study, under-expression of GPX3 was associated with metastasis of thyroid cancer. Moreover, expression levels of GPX3 corresponded with stage of the cancer ${ }^{[27]}$. In a related study, ${ }^{[28]}$ under expression of GPX3 was associated with larger volume, more nodules worse clinical stage and poor prognosis of HCC. Both in vivo and in vitro studies ${ }^{\text {[29] }}$ demonstrated that underexpression of GPX3 participated in the invasion and metastasis of gastric cancer. In this study, we found dysregulated GPX3 expression in 26 of 33 tumor types. Particularly, GPX3 expression was downregulated in 22 
but up-regulated in 4 of the tumor types. Further analyses revealed under expression of GPX3 protein in all 6 tumors in CPTAC database. We also found GPX3 expression correlated with pathological stages of tumors. Overall, GPX3 expression significantly impacts on progression of several tumors.

GPX3 expression levels influences the prognosis of most tumors. However, there are different reports about the role of GPX3 high / low expression in different tumor prognosis. Several studies have shown that loss of GPX3 expression in tumor tissues is associated with poor prognosis and chemotherapy resistance in patients ${ }^{[30,31]}$. Low expression of GPX3 can also predict patient prognosis. For instance, Caroline $\mathrm{C}$ et al. ${ }^{[32]}$ found that underexpression of GPX3 strongly correlated with low survival rate of lung adenocarcinoma and low-grade gliomas. However, GPX3 expression was elevated in other tumor tissues ${ }^{[33,34,35]}$, high expression of GPX3 is associated with poor prognosis in cancer patients such as gastric cancer and lung squamous cell carcinoma. Herein, tumor patients were divided into high and low expression GPX3 expression groups. We found low-expression of GPX3 resulted in better prognosis of patients with BLCA, COAD and STAD, but poor prognosis of PAAD. Meanwhile, lowexpression of GPX3 was linked to longer DFI and PFI of patients with BRCA, LUAD, STAD, COAD and STAD but poor PFI of PAAD patients. Further Univariate Cox regression analyses revealed that GPX3 expression levels predicted the OS, DFS, PFS and DSS of patients with several cancers such as PAAD, STAD, BRCA, LUAD and COAD. It can be concluded that in OS, DFS and PFS, PAAD with low GPX3 expression has a worse prognosis, and COAD and STAD with low GPX3 expression have a better prognosis. When GPX3 expression was low in STAD and BRCA, DSS was longer, whereas LUAD was shorter. That is, although Fig. 4 and Fig. 5 were different algorithms, they both illustrate the prognostic value of GPX3 in different tumors. It can be seen that GPX3 plays a dichotomy role in different tumor types, both as a tumor suppressor protein and as a survival promoting protein. However, it is necessary to further study the molecular evidence of how the high / low expression of GPX3 specifically affects the scavenging and redox signals of oxidants in the microenvironment of tumor cells.

This presents the first report of the association between GPX3 expression and TMB/MSI, and some tumor types that may benefit more from immunotherapy were identified. The potential relationship between infiltration of Immune cell and GPX3 expression in different tumor types was understood. This study also showed that GPX3 expression positively correlated with infiltration of CAFs in BLCA, BRCA, BRCA-Basal, etc. Besides, GPX3-binding components and GPX3 expression related genes in all TCGA tumors were integrated for enrichment analysis, and it was found that "AMPK signaling pathway" and "Fructose and mannose metabolism" had an influence on the etiology or pathogenesis of tumors. The nomograms were constructed and validated to provide a prediction of 1-, 2-, 3- and 5-year survival in patients with STAD. It showed good performance in applicability and accuracy, which also supported that the relationship between GPX3 expression and STAD discovered above. Combined with the existing research results, we speculate that GPX3 plays an important role in the occurrence and development of STAD, and is expected to become a new target for the diagnosis and treatment of STAD. However, due to the small sample size in this study, more experiments are needed to explore the specific mechanism of GPX3 in the development of STAD and other cancers.

\section{Conclusion}

In summary, the mRNA level of GPX3 is altered in 26 types of malignant tumors. Specifically, it is upregulated in 4 types of tumors and downregulated in 22 types of tumors, suggesting that the low expression of GPX3 contributes to the occurrence and development of some tumors. Changes in GPX3 expression can affect the OS, DFS, PFS, DFI, PFI and DSS of 3 types of tumors. In all TCGA tumors, the potential correlation between GPX3

Page $10 / 26$ 
expression and MSI or TMB was proposed for the first time. The expression of GPX3 in most tumors was positively correlated with the infiltration level of 6 classic immune cells. Meanwhile, the expression of GPX3 in some tumors is related to the level of immune infiltration of CAFs. GPX3 participates in tumorigenesis by regulating AMPK signaling pathway. After functional validation and prognostic modeling, GPX3 was found to be a prognostic marker for STAD. This study provides blueprint data concerning the correlation between GPX3 and human tumors. The results of this analysis therefore expand our understanding on the role and mechanism of $G P X 3$ in tumorigenesis.

\section{Abbreviations}

$G P X$

Glutathione peroxidase

ROS

Reactive oxygen species

GPX3

Glutathione peroxidase 3

TCGA

The Cancer Genome Atlas

HPA

Human Protein Atlas

$\mathrm{IHC}$

immunohistochemical

TPM

Transcripts per million

CPTAC

Clinical proteomic tumor analysis consortium

GEO

Gene Expression Omnibus

Clear cell RCC

Clear cell Renal Cell Carcinoma

UCEC

Uterine Corpus Endometrial Carcinoma

LUAD

Lung adenocarcinoma

OS

Overall Survival

DFS

Disease-Free Survival

PFS

Progression Free Survival

DSS

Disease Specific Survival

DFI 
Disease-Free Interval

PFI

Progression-Free Interval

$\mathrm{HR}$

hazard ratios

$\mathrm{Cl}$

confidence interval

GDC

genomic data Commons

TMB

Tumor Mutation Burden

MSI

Microsatellite Instability

CAFs

Cancer associated fibroblasts

TME

tumor microenvironment

PCC

Pearson's correlation coefficient

PPI

Protein-protein interaction

KEGG

Kyoto Encyclopedia of Genes and Genomes

GO

Gene Ontology

BP

Biological Process

CC

Cellular Component

MF

Molecular Function

C-index

Concordance index

ACC

Adrenocortical carcinoma

BLCA

Bladder Urothelial Carcinoma

BRCA

Breast invasive carcinoma

CESC

Cervical squamous cell carcinoma and endocervical adenocarcinoma $\mathrm{CHOL}$

Cholangiocarcinoma, COAD:Colon adenocarcinoma 
ESCA

Esophageal carcinoma, HNSC:Head and Neck squamous cell carcinoma

$\mathrm{KICH}$

Kidney Chromophobe

KIRC

Kidney renal clear cell carcinoma

KIRP

Kidney renal papillary cell carcinoma

LUAD

Lung adenocarcinoma

LUSC

Lung squamous cell carcinoma

OV

Ovarian serous cystadenocarcinoma

PRAD

Prostate adenocarcinoma

READ

Rectum adenocarcinoma

SKCM

Skin Cutaneous Melanoma

STAD

Stomach adenocarcinoma

TGCT

Testicular Germ Cell Tumors

THCA

Thyroid carcinoma

UCEC

Uterine Corpus Endometrial Carcinoma

UCS

Uterine Carcinosarcoma

DLBC

Lymphoid Neoplasm Diffuse Large B-cell Lymphoma

GBM

Glioblastoma multiforme

LGG

Glioma

LIHC

Liver hepatocellular carcinoma

PAAD

Pancreatic adenocarcinoma

LAML

Acute Myeloid Leukemia

UVM 
Uveal Melanoma

MESO

Mesothelioma

PCPG

Pheochromocytoma and Paraganglioma

SARC

Sarcoma

THYM

Thymoma

AUC

Area Under Curve.

\section{Declarations}

\section{Ethical Approval and Consent to Participate}

All data for this study are derived from publicly available databases and does not directly involve human participants. Therefore, ethical approval was not required for this article.

\section{Consent for publication}

Not relevant.

\section{Availability of data and materials}

The TCGA and GTEx data set was obtained from open databases. TCGA data set can be obtained from the following website: http://cancergenome.nih.gov. GTEx data set can be obtained from the following website: http://commonfund.nih.gov/GTEx/.

\section{Competing Interests}

The authors declare no conflict of interest.

\section{Funding}

This study was supported by Key Research and Development Program in Shaanxi Province (2020SF-076), Research Project from Health Commission of Shaanxi Provincial Government (2018A017), Education Department of Shaanxi Provincial Government (19JS015), Special R囚D Program Project of Chinese Academy of Se-enriched Industry (2020FXZX05-01), Chinese Medicine Science and Technology Research Special Project of State Administration of Traditional Chinese Medicine (GZY-KJS-2021-006), National Science Foundation Project (81603454), Clinical collaborative innovation project of integrated traditional Chinese and Western medicine by Shaanxi Administration of traditional Chinese Medicine (2020-ZXY-005), Shaanxi Province Chinese Medicine Young and Middle-aged Science and Technology Key Talents Project, National Key Talents Project of Traditional Chinese Medicine Clinical Characteristic Technology Inheritance.

\section{Authors' contributions}


Study design and conception: Rongqiang Zhang, Yuetong Wang

Data acquisition: Yuetong Wang, Kai Qu

Analysis and interpretation of data: Rongqiang Zhang, Yuetong Wang

All authors read and revised the manuscript, and approved the final version to be published.

\section{Acknowledgments}

The authors thank the patients and their families, who have generously donated their organizations to the TCGA, and thank the TCGA members for collecting and disclosing

valuable data.

\section{Authors' information}

NA

\section{References}

[1] Lin GW, Wang JY, Ge JB. Practice of Internal Medicine. Beijing: People's Medical Publishing House; 2017.170.

[2] World Health Organization International Agency for Research on Cancer: Latest global cancer data: Cancer burden rises to 19.29 million new cases and 9.96 million cancer deaths in 2020

[3] Zhang J, Wang S, Meng FJ, Yan YJ, Wang B, Guan ZY. Research progress of glutathione peroxidase in tumor. Chinese Journal of Cancer 2019; 38: 282-287.

[4] Jiang YF, Qiu BX, Pang BY, Zhang YF. Research progress on the relationship between GPX3 and tumor and its mechanism. Guangdong Medical Journal 2018; 39: 258-260+265.

[5] Kalatskaya I. Overview of major molecular alterations during progression from Barrett's esophagus to esophageal adenocarcinoma. Ann N Y Acad Sci 2016; 1381: 74-91. https://doi.org/10.1111/nyas.13134

[6] Fujiwara S, Nagai H, Jimbo H, Jimbo N, Tanaka T, Inoie M, et al. Gene Expression and Methylation Analysis in Melanomas and Melanocytes from the Same Patient: Loss of NPM2 Expression Is a Potential Immunohistochemical Marker for Melanoma. Front Oncol 2019; 8: 675. https://doi.org/10.3389/fonc.2018.00675

[7] Hughes DJ, Kunická T, Schomburg L, Liška V, Swan N, Souček P. Expression of Selenoprotein Genes and Association with Selenium Status in Colorectal Adenoma and Colorectal Cancer. Nutrients 2018; 10 : 1812. https://doi.org/10.3390/nu10111812

[8] Zhou C, Pan R, Li B, Huang T, Zhao J, Ying J, et al. GPX3 hypermethylation in gastric cancer and its prognostic value in patients aged over 60. Future Oncol 2019; 15: 1279-1289. https://doi.org/10.2217/fon-2018-0674

[9] Worley BL, Kim YS, Mardini J, Zaman R, Leon KE, Vallur PG, et al. GPx3 supports ovarian cancer progression by manipulating the extracellular redox environment. Redox Biol 2019; 25: 101051.

https://doi.org/10.1016/j.redox.2018.11.009

Page 15/26 
[10] Shen SX (2020) Analysis of the expression characteristics of pepsinogen $C$ gene in pan-cancer and its regulatory mechanism. [dissertation/master's thesis]. [Liao Ning]: China Medical University.

[11] Zhang K, Wang H. Cancer Genome Atlas Pan-cancer Analysis Project. Zhongguo Fei Ai Za Zhi 2015; 18:21923. https://doi.org/10.3779/j.issn.1009-3419.2015.04.02

[12] Ye W, Luo C, Liu F, Liu Z, Chen F. CD96 Correlates with Immune Infiltration and Impacts Patient Prognosis: A Pan-Cancer Analysis. Front Oncol 2021; 11: 634617. https://doi.org/10.3389/fonc.2021.634617

[13] Tang Z, Kang B, Li C, Chen T, Zhang Z. GEPIA2: an enhanced web server for large-scale expression profiling and interactive analysis. Nucleic Acids Res 2019; 47: W556-W560. https://doi.org/10.1093/nar/gkz430

[14] Chen F, Chandrashekar DS, Varambally S, Creighton CJ. Pan-cancer molecular subtypes revealed by massspectrometry-based proteomic characterization of more than 500 human cancers. Nat Commun 2019; 10 : 5679. https://doi.org/10.1038/s41467-019-13528-0

[15] Cui X, Zhang X, Liu M, Zhao C, Zhang N, Ren Y, et al. A pan-cancer analysis of the oncogenic role of staphylococcal nuclease domain-containing protein 1 (SND1) in human tumors. Genomics 2020; 112: 3958-3967. https://doi.org/10.1016/j.ygeno.2020.06.044

[16] Ju Q, Li XM, Zhang H, Zhao YJ. BRCA1-Associated Protein Is a Potential Prognostic Biomarker and Is Correlated With Immune Infiltration in Liver Hepatocellular Carcinoma: A Pan-Cancer Analysis. Front Mol Biosci 2020; 7: 573619. https://doi.org/10.3389/fmolb.2020.573619

[17] Thorsson V, Gibbs DL, Brown SD, Wolf D, Bortone DS, Ou Yang TH, et al. The Immune Landscape of Cancer. Immunity 2018; 48: 812-830.e14. https://doi.org/10.1016/j.immuni.2018.03.023

[18] Bonneville R, Krook MA, Kautto EA, Miya J, Wing MR, Chen HZ, et al. Landscape of Microsatellite Instability Across 39 Cancer Types. JCO Precis Oncol 2017; 2017: P0.17.00073. https://doi.org/10.1200/P0.17.00073

[19] Pan JH, Zhou H, Cooper L, Huang JL, Zhu SB, Zhao XX, et al. LAYN Is a Prognostic Biomarker and Correlated With Immune Infiltrates in Gastric and Colon Cancers. Front Immunol 2019; $10: 6$.

https://doi.org/10.3389/fimmu.2019.00006

[20] Wang Z, Gao L, Guo X, Feng C, Lian W, Deng K, et al. Development and validation of a nomogram with an autophagy-related gene signature for predicting survival in patients with glioblastoma. Aging (Albany NY) 2019; 11: 12246-12269. https://doi.org/10.18632/aging.102566

[21] Chen L, Long C, Liu J, Duan X, Xiang Z. Prognostic nomograms to predict overall survival and cancer-specific survival in patients with pelvic chondrosarcoma. Cancer Med 2019; 8: 5438-5449.

https://doi.org/10.1002/cam4.2452

[22] Chang QH, Mao T, Tao Y, Dong T, Tang XX, Ge GH, et al. Pan-cancer analysis identifies ITIH1 as a novel prognostic indicator for hepatocellular carcinoma. Aging (Albany NY) 2021; 13:11096-11119. https://doi.org/10.18632/aging.202765 
[23] Chen M, Zhang S, Nie Z, Wen X, Gao Y. Identification of an Autophagy-Related Prognostic Signature for Clear Cell Renal Cell Carcinoma. Front Oncol 2020;10: 873. https://doi.org/10.3389/fonc.2020.00873

[24] Saelee P, Pongtheerat T, Sophonnithiprasert T. Reduced Expression of GPX3 in Breast Cancer Patients in Correlation with Clinical Significance. Glob Med Genet 2020; 7: 87-91. https://doi.org/10.1055/s-0040-1722170

[25] Lou W, Ding B, Wang S, Fu P. Overexpression of GPX3, a potential biomarker for diagnosis and prognosis of breast cancer, inhibits progression of breast cancer cells in vitro. Cancer Cell Int 2020; 20: 378.

https://doi.org/10.1186/s12935-020-01466-7

[26] Noci S, Dugo M, Bertola F, Melotti F, Vannelli A, Dragani TA, et al. A subset of genetic susceptibility variants for colorectal cancer also has prognostic value. Pharmacogenomics J 2016; 16: 173-9.

https://doi.org/10.1038/tpj.2015.35

[27] Li HL, Yu W, Chen YT, Li J. Changes of serum Gal-1 and GPX3 expression in patients with thyroid cancer and their relationship with disease severity 2020; 22: 191-193.

[28] Wang Y, Zhang WY, Han QW, Liu Y, Dai HR, Guo YL, et al. Effective response and delayed toxicities of refractory advanced diffuse large B cell lymphoma treated by CD20 directed chimeric antigen receptor modified $T$ cells. Clin Immunol 2014; 155: 160-175. https://doi.org/10.1016/j.clim.2014.10.002

[29] Qu Y, Dang S, Hou P. Gene methylation in gastric cancer. Clin Chim Acta 2013; 424: 53-65. https://doi.org/10.1016/j.cca.2013.05.002

[30] An BC, Choi YD, Oh IJ, Kim JH, Park JI, Lee SW. GPx3-mediated redox signaling arrests the cell cycle and acts as a tumor suppressor in lung cancer cell lines. PLoS One 2018; 13: e0204170.

https://doi.org/10.1371/journal.pone.0204170

[31] Cai M, Sikong Y, Wang Q, Zhu S, Pang F, Cui X. Gpx3 prevents migration and invasion in gastric cancer by targeting NFKB/Wnt5a/JNK signaling. Int J Clin Exp Pathol 2019; 12:1194-1203.

[32] Chang C, Worley BL, Phaëton R, Hempel N. Extracellular Glutathione Peroxidase GPx3 and Its Role in Cancer. Cancers (Basel) 2020; 12: 2197. https://doi.org/10.3390/cancers12082197

[33] Miess H, Dankworth B, Gouw AM, Rosenfeldt M, Schmitz W, Jiang M, et al. The glutathione redox system is essential to prevent ferroptosis caused by impaired lipid metabolism in clear cell renal cell carcinoma. Oncogene 2018; 37:5435-5450. https://doi.org/10.1038/s41388-018-0315-z

[34] Lee HJ, Do JH, Bae S, Yang S, Zhang X, Lee A, et al. Immunohistochemical evidence for the over-expression of Glutathione peroxidase 3 in clear cell type ovarian adenocarcinoma. Med Oncol 2011;28 Suppl 1: S522-7. https://doi.org/10.1007/s12032-010-9659-0

[35] Pelosof L, Yerram S, Armstrong T, Chu N, Danilova L, Yanagisawa B, et al. GPX3 promoter methylation predicts platinum sensitivity in colorectal cancer. Epigenetics 2017; 12: 540-550.

https://doi.org/10.1080/15592294.2016.1265711

\section{Figures}

Page $17 / 26$ 

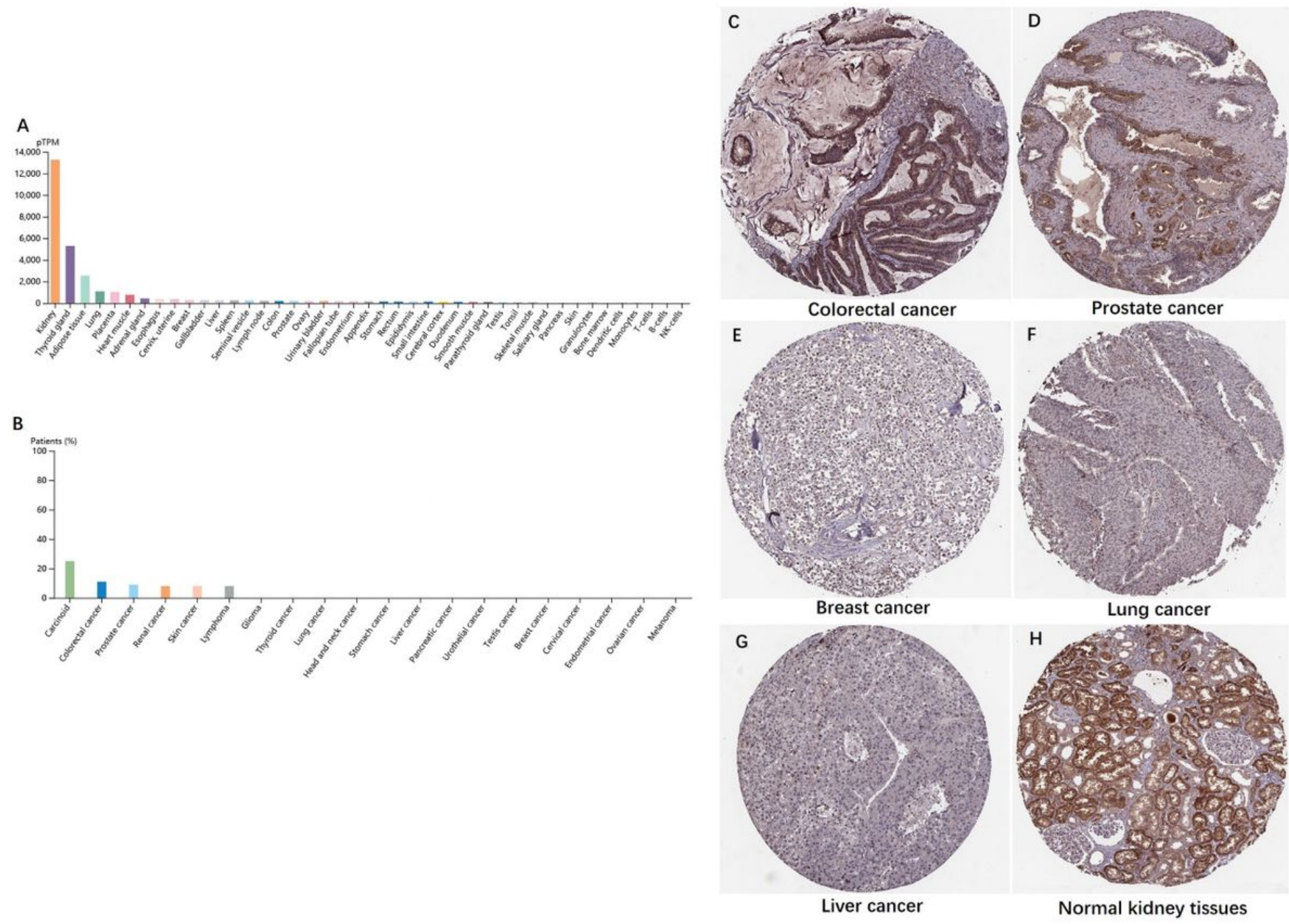

\section{Figure 1}

Expression profiles of GPX3 in normal and cancer tissues. (A) The mRNA level of GPX3 in normal human tissues [Y-axis means protein-coding transcripts per million (pTPM); X-axis means normal human tissue]. (B) Detection rate of GPX3 protein in human tumor tissues. (C-H) Representative IHC images showing GPX3 expression in colorectal cancer, prostate cancer, breast cancer, lung cancer, liver cancer and normal kidney tissues. 

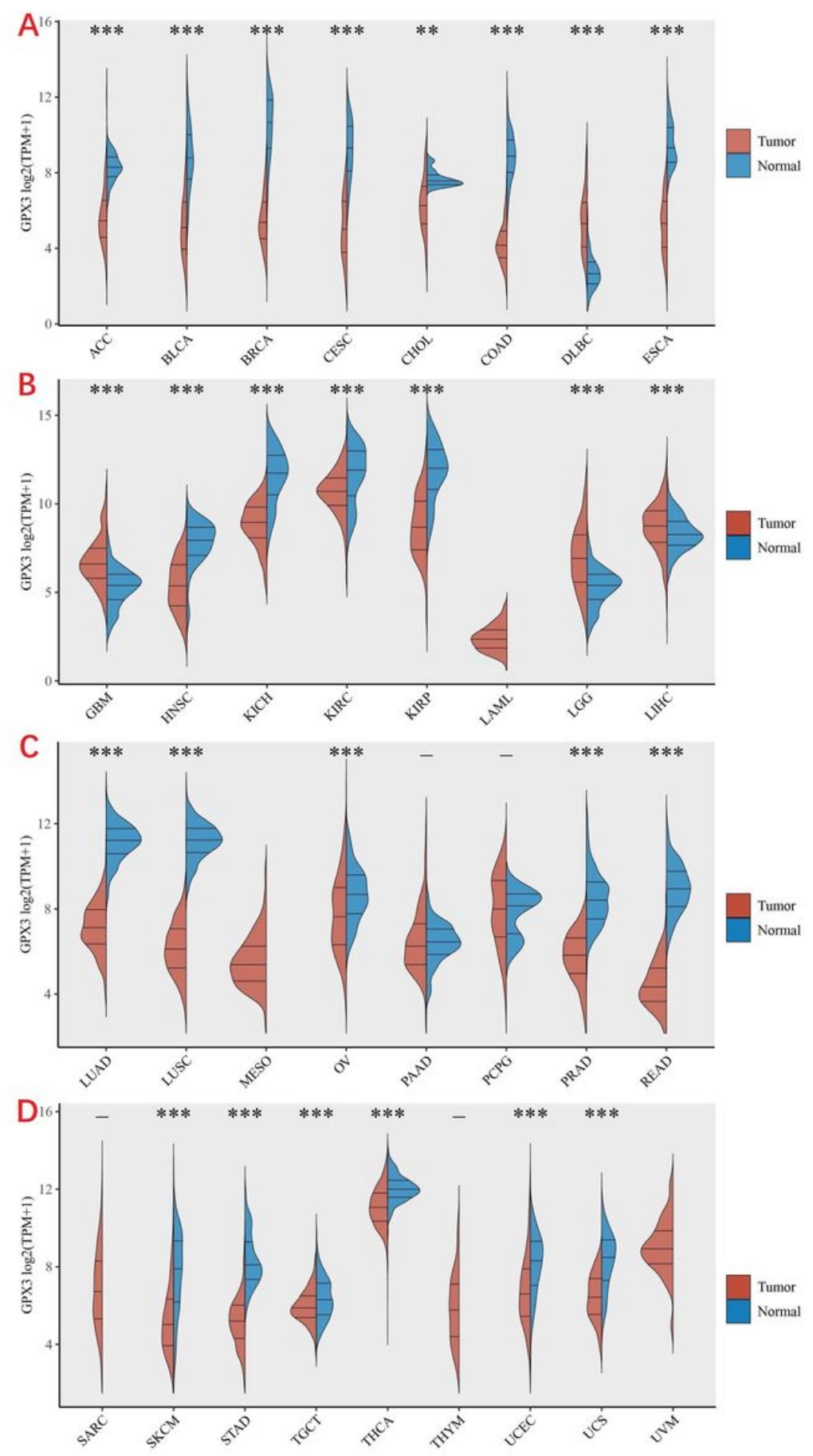

TCGA+GTEx database

Figure 2

Expression level of GPX3 gene in different cancers from TCGA+GTEx database $\left({ }^{\star} P<0.05 \rrbracket^{\star \star} P<0.01 \rrbracket \star \star \star P<0.001\right)$ 


\section{A CPTAC dataset}
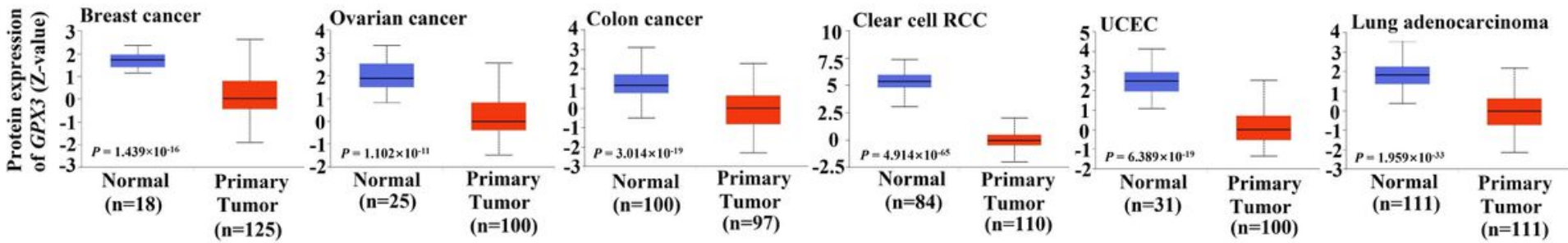

\section{B TCGA dataset}
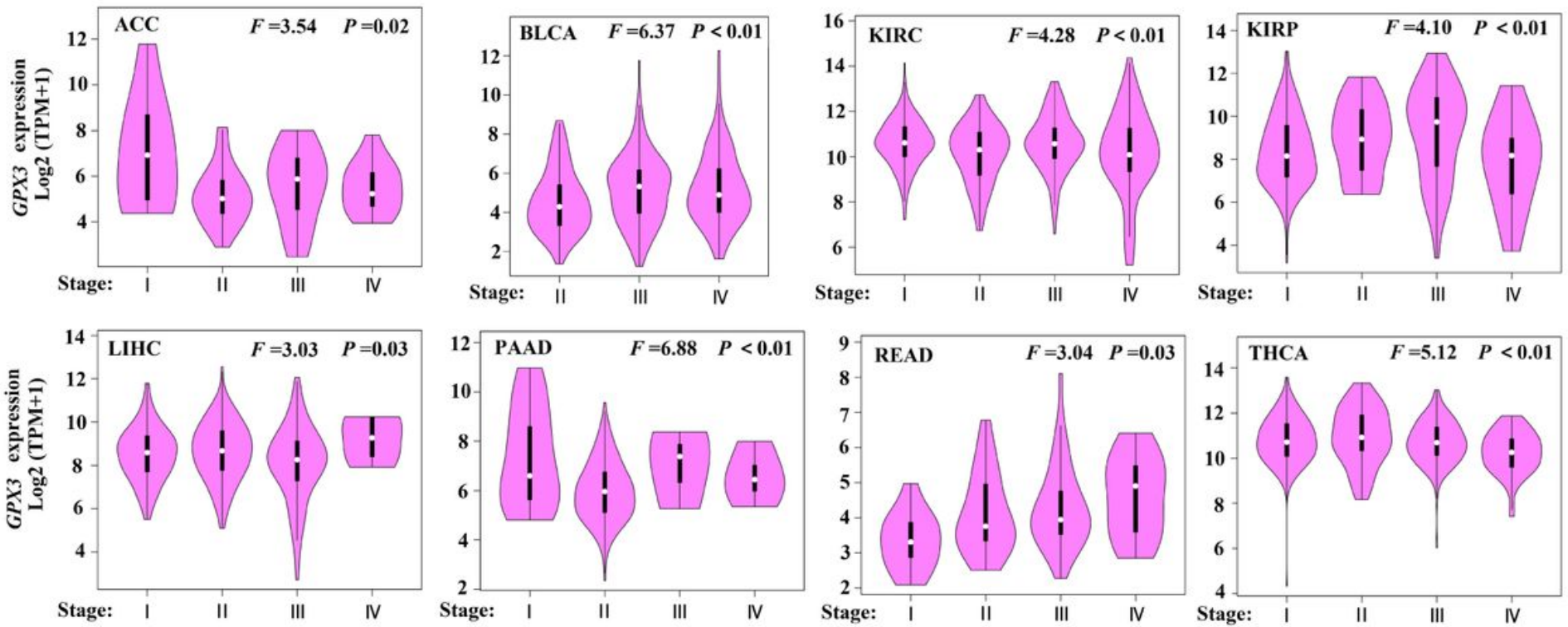

Figure 3

GPX3 expression level in different cancers (A) Expression level of GPX3 total protein in normal tissues and primary tumors of breast cancer, ovarian cancer, colon cancer, clear cell RCC, UCEC, lung adenocarcinoma. (B) Expression level of GPX3 in the main pathological stages of ACC, BLCA, KIRC, KIRP, LIHC, PAAD, READ and THCA.

A

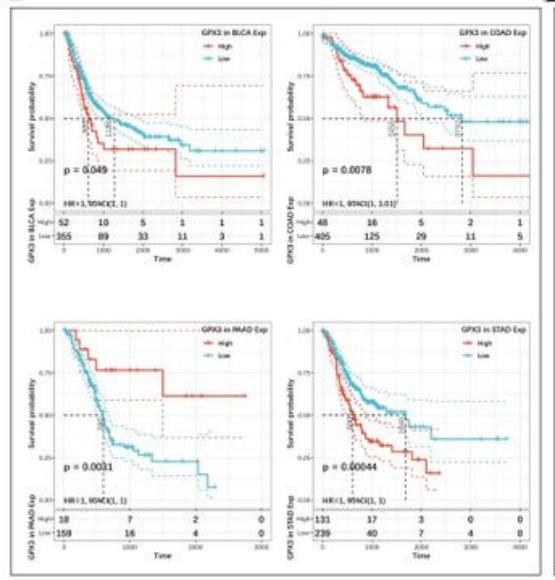

B
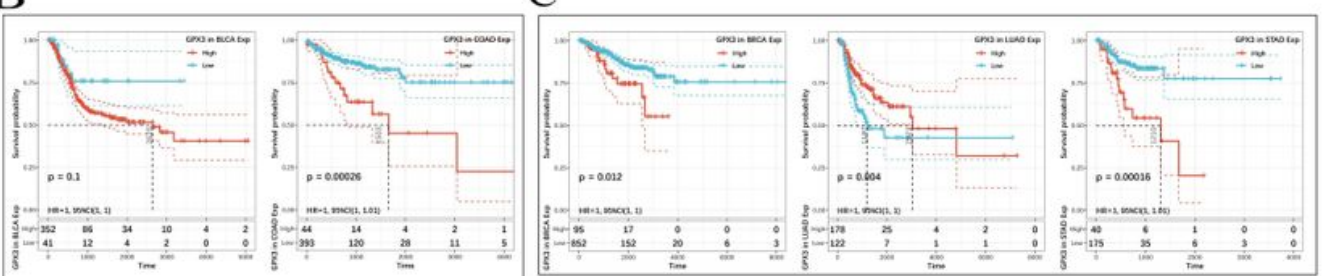

$\mathrm{D}$

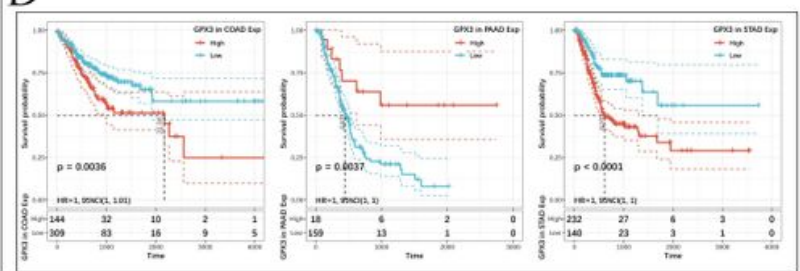

Figure 4 
Kaplan-Meier curves reflect the prognostic value of GPX3 in different cancers (A) OS of patients in BLCA, COAD, PAAD and STAD with low or high GPX3 (B) DSS of patients in BLCA, COAD, PAAD and STAD with low or high GPX3. (C) Kaplan-Meier DFI curves for patients stratified by different expression levels of GPX3 in BLCA, LUAD and STAD. (D) PFI of patients in COAD, PAAD and STAD with low or high GPX3.
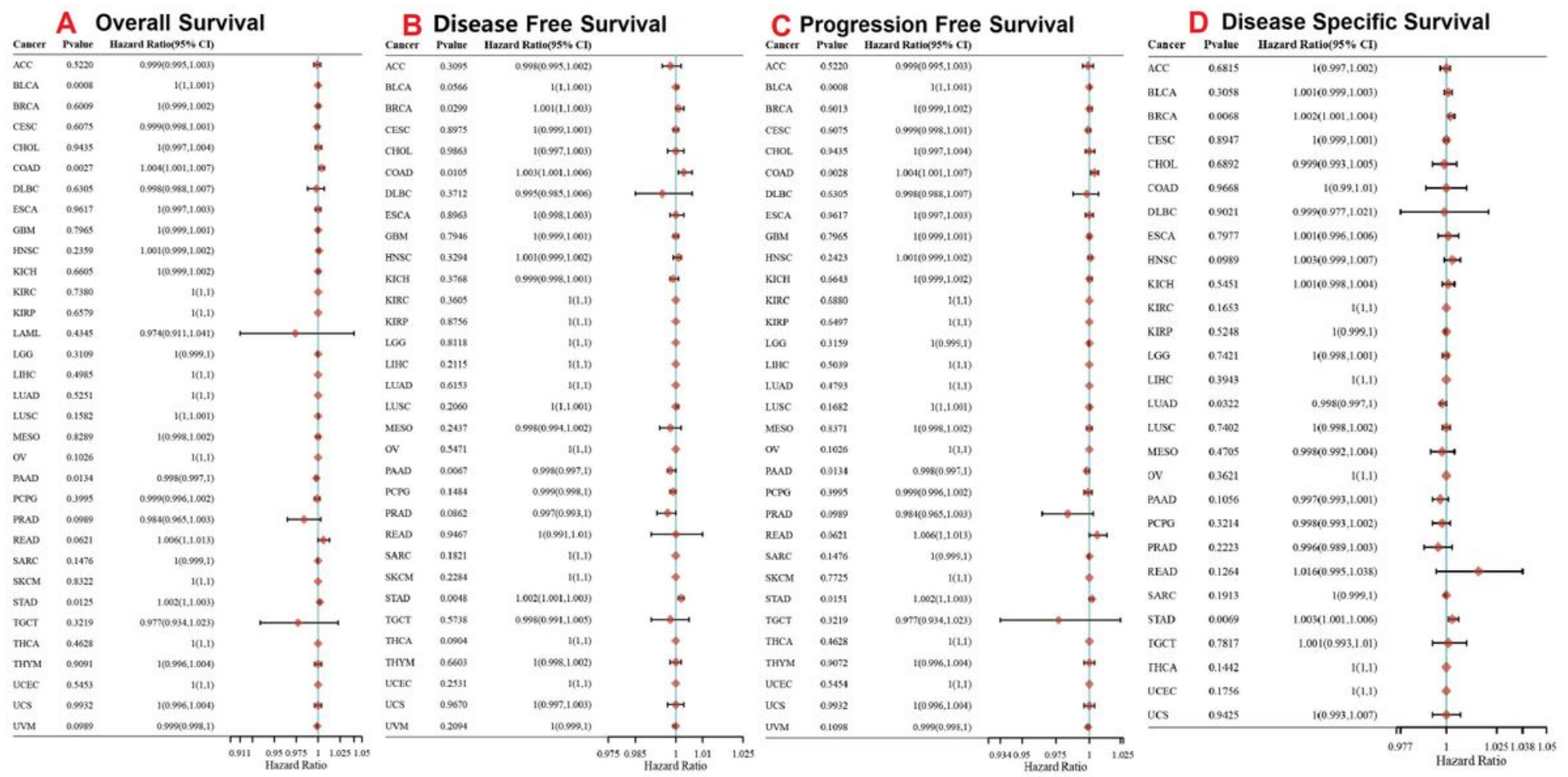

\section{Figure 5}

Correlation of GPX3 gene expression with patient's OS, DFS, PFS and DSS in different cancer types. The forest plots with the hazard ratios (HR) and 95\% confidence intervals for OS, DFS, PFS and DSS in different cancers showing the survival advantage and disadvantage of low expression of GPX3. (HR>1, indicates that high GPX3 expression predicts worse prognosis compared to low GPX3 expression, whereas HR< 1 indicates that high GPX3 expression predicts better prognosis than low GPX3 expression) 


\section{A TMB}

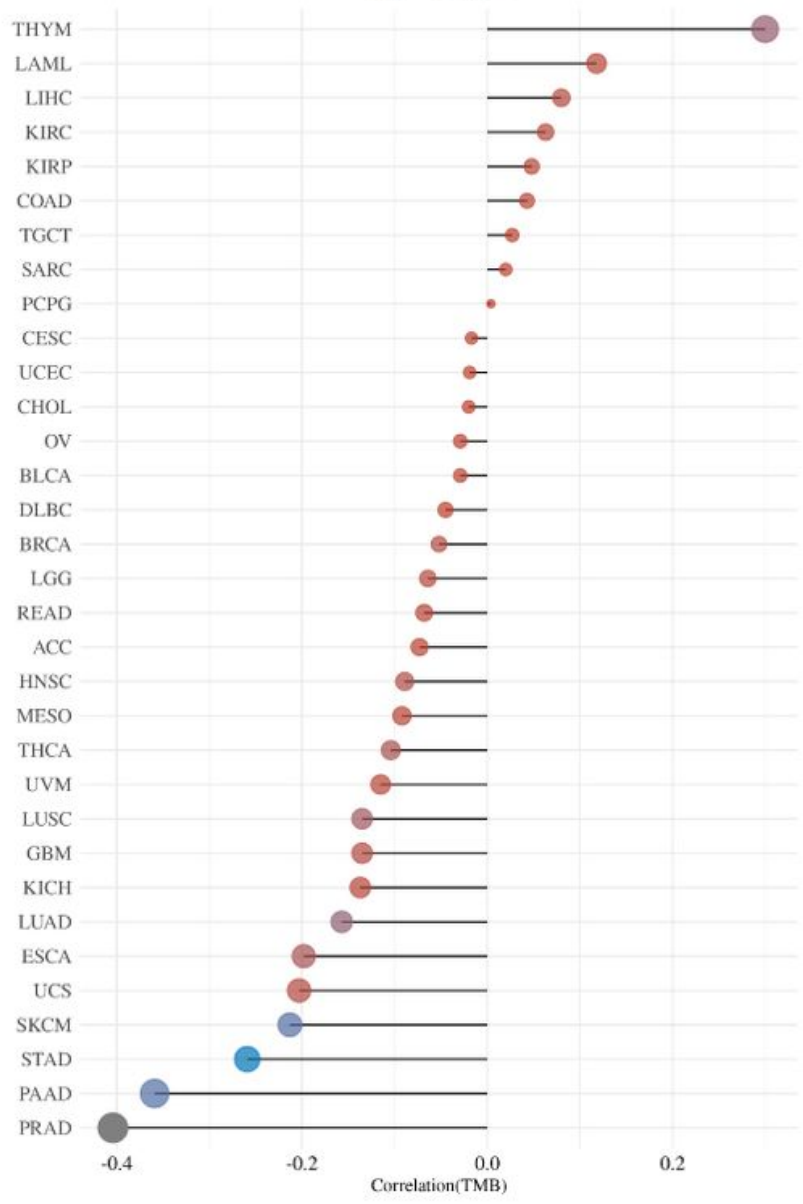

\section{B MSI}

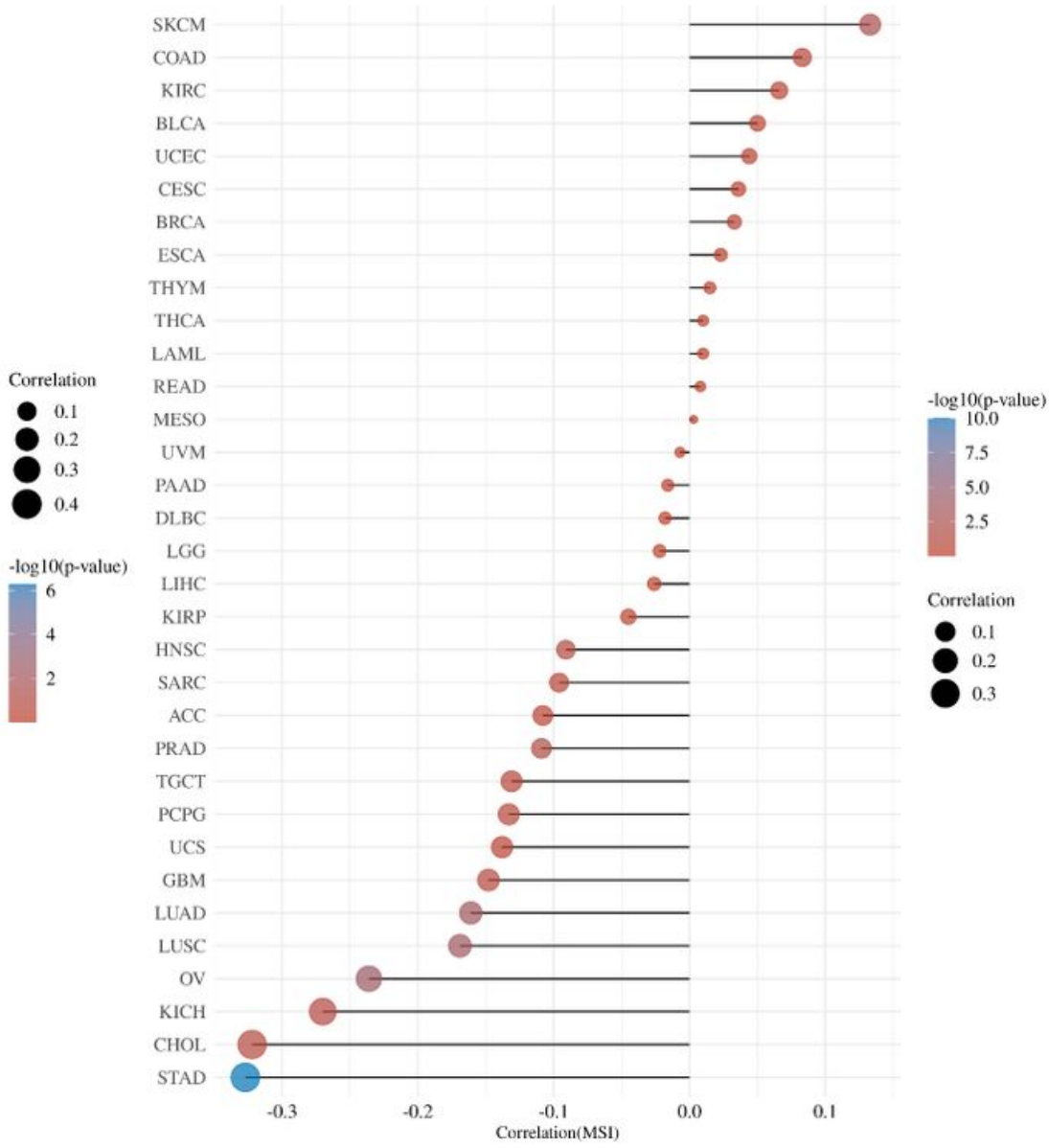

\section{Figure 6}

Mutation profiles of GPX3 in different cancers from the TCGA (A) Spearman correlation analysis showing the association between TMB and GPX3 gene expression (B) Spearman correlation analysis showing the correlation between MSI and GPX3 gene expression (The X-axis represents the correlation coefficient between genes and $\mathrm{TMB} / \mathrm{MSI}$, while the $\mathrm{Y}$-axis represents different tumors. The size of dots in the figure represents the correlation coefficient, and different colors represent the significance of $\mathrm{P}$ value. In the schematic diagram, the redder the color, the smaller the $\mathrm{P}$ value) 
A

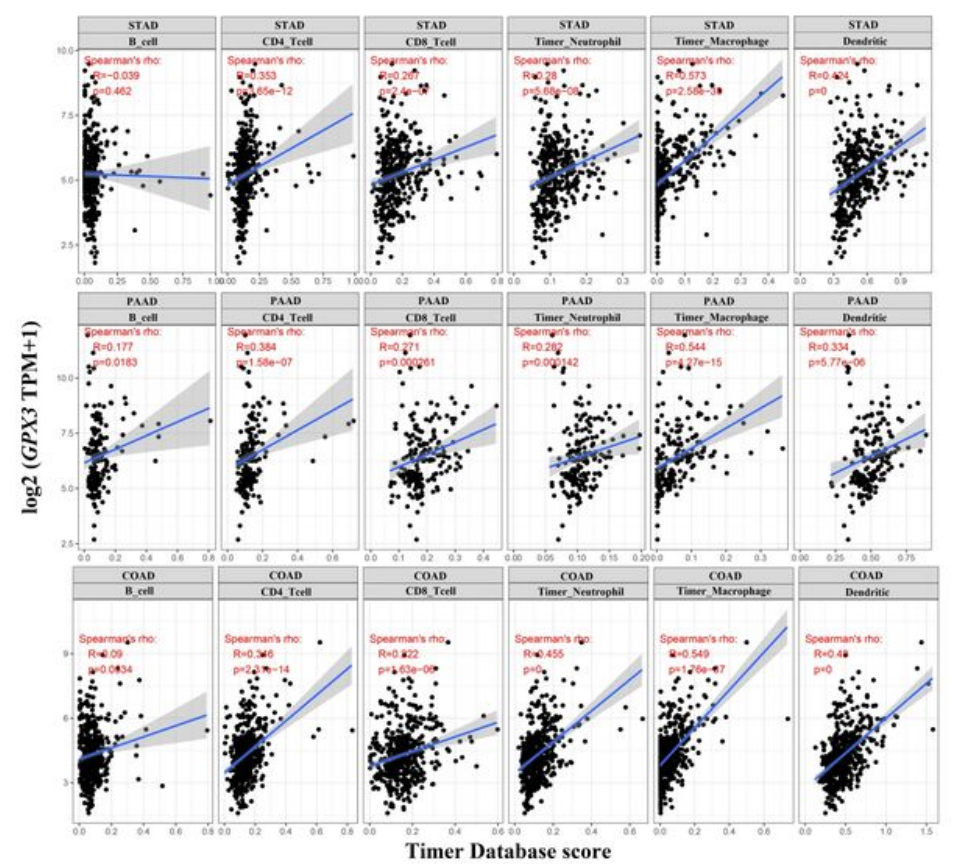

B

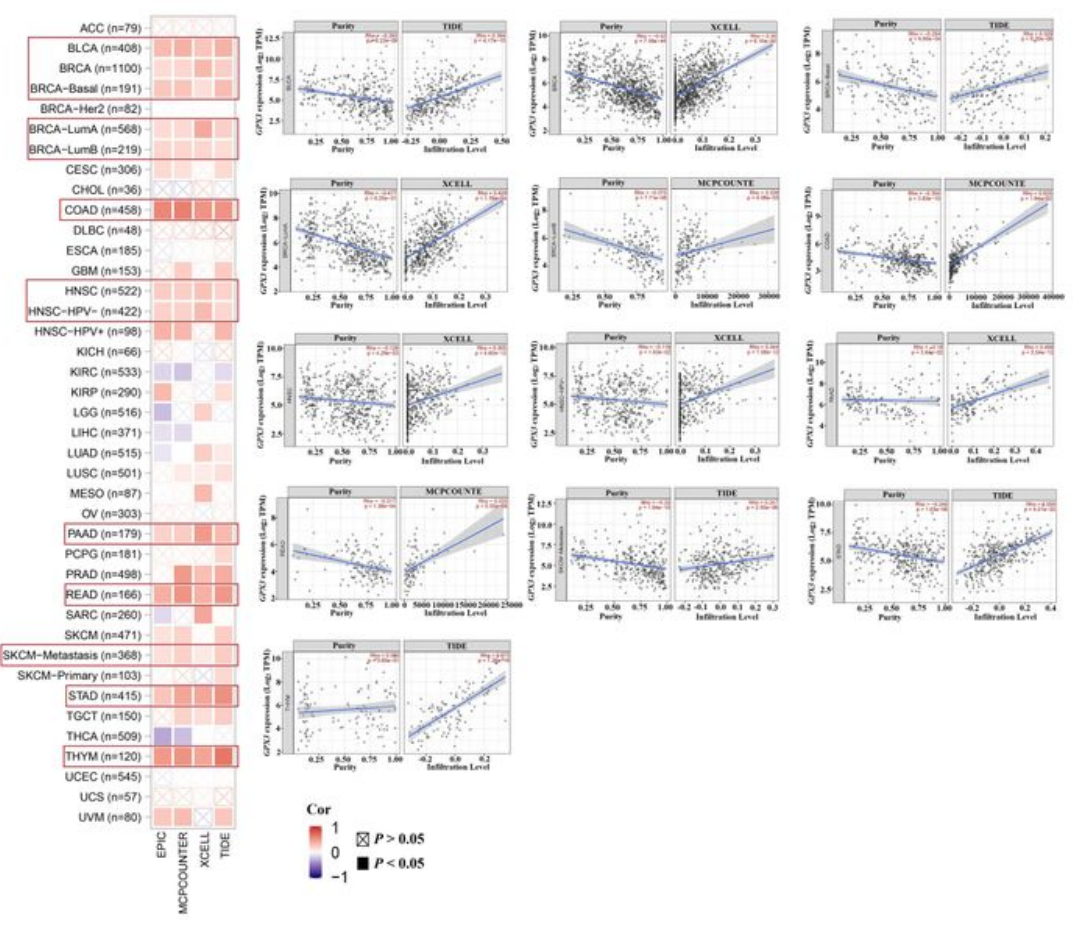

\section{Figure 7}

Correlation analysis between GPX3 expression and immune infiltration (A) Correlation between GPX3 expression and the level of STAD, PAAD and COAD immune infiltration (B) Correlation between the expression of GPX3 and immune infiltration of CAFs. 
A
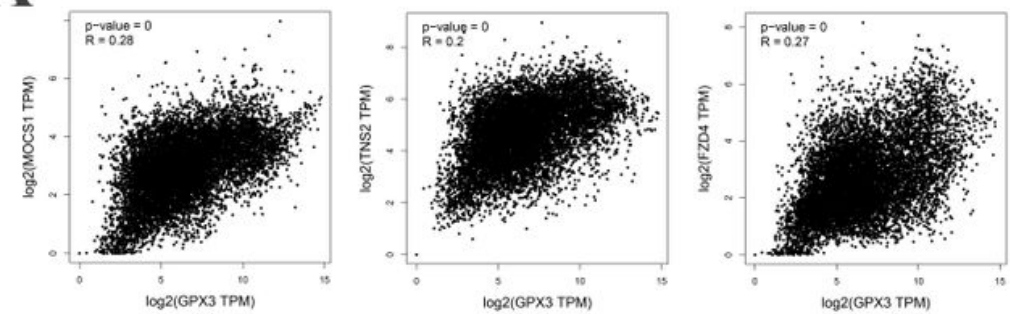

C

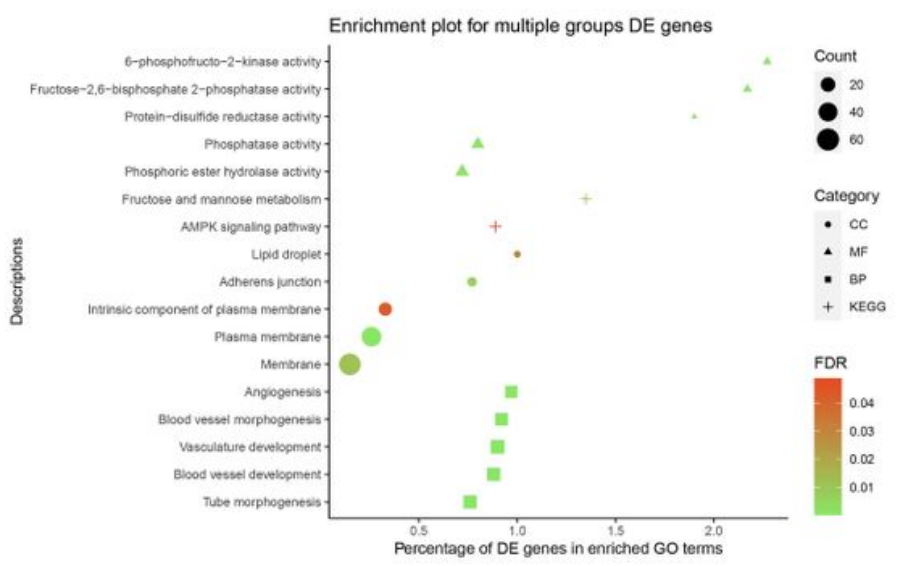

B

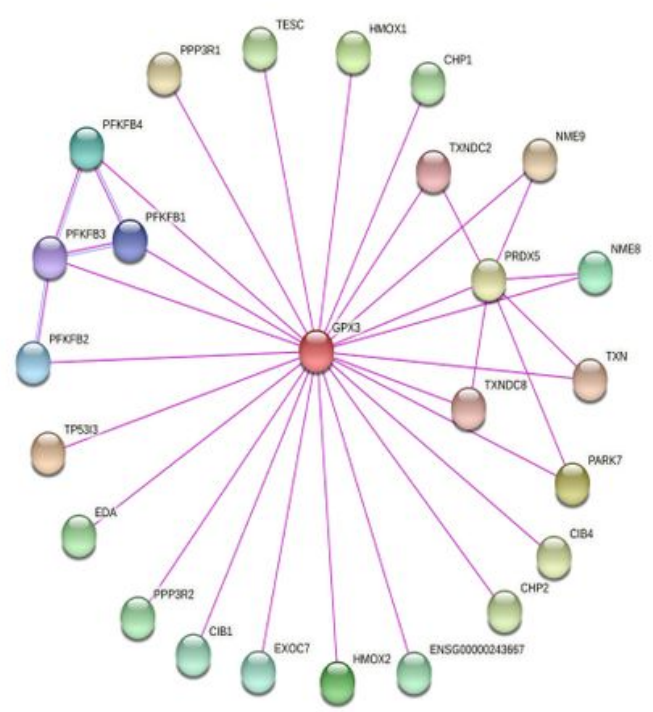

Figure 8

Enrichment gene associated with GPX3 (A) The correlation between GPX3 and the top 3 similarly expressed genes (B) A protein-protein interaction network for GPX3 (C) The results of enrichment analysis for GPX3 in different human tumors. 
A COAD
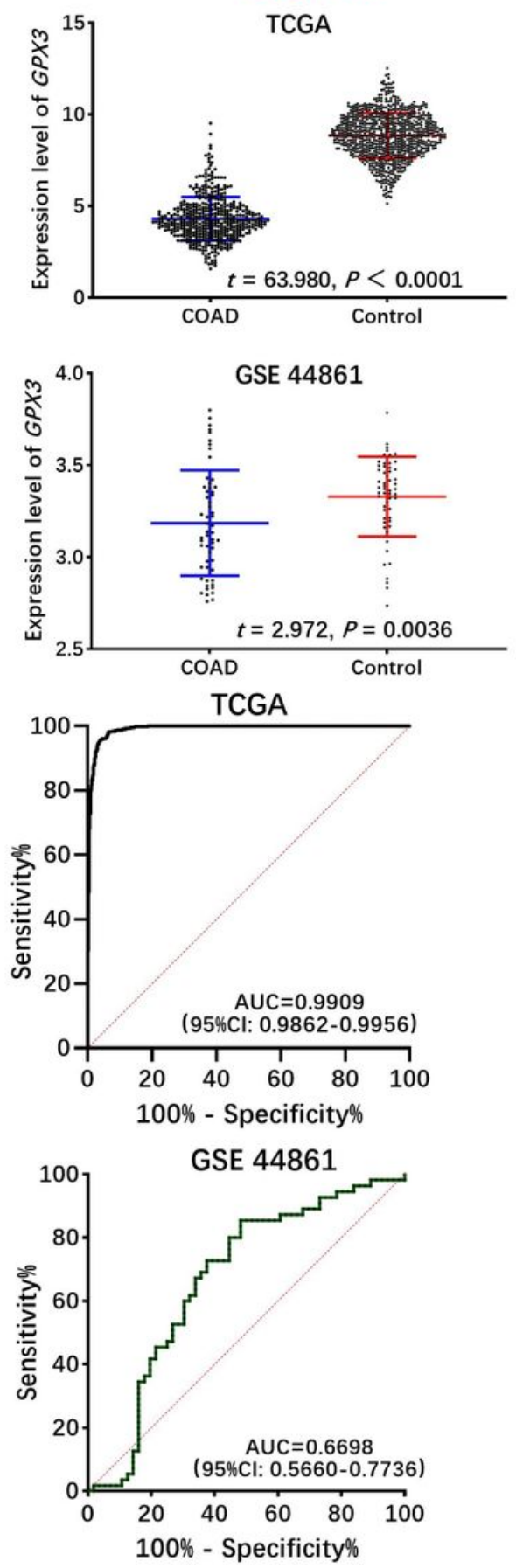

B STAD
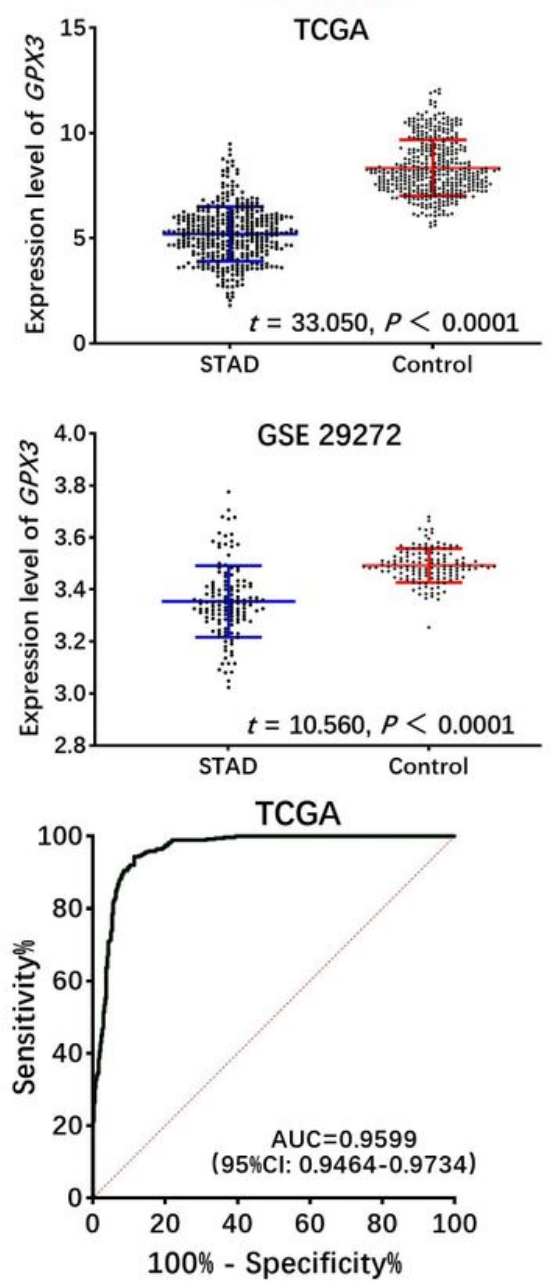

GSE 29272

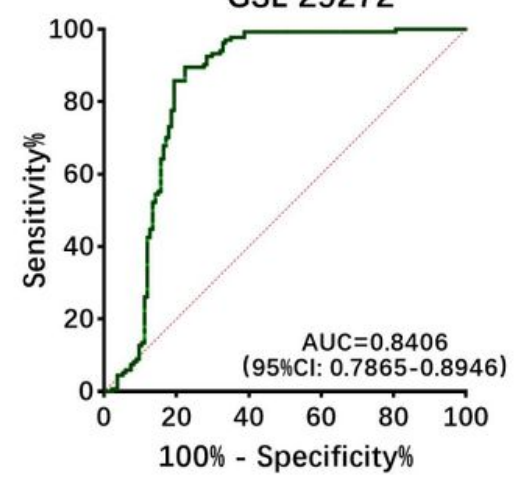

\section{Figure 9}

Independent validation of the differential expression and prognostic significance of GPX3 in GEO and TCGA datasets (A) the differential expression and prognostic significance of GPX3 in COAD (B) the differential expression and prognostic significance of GPX3 in STAD 

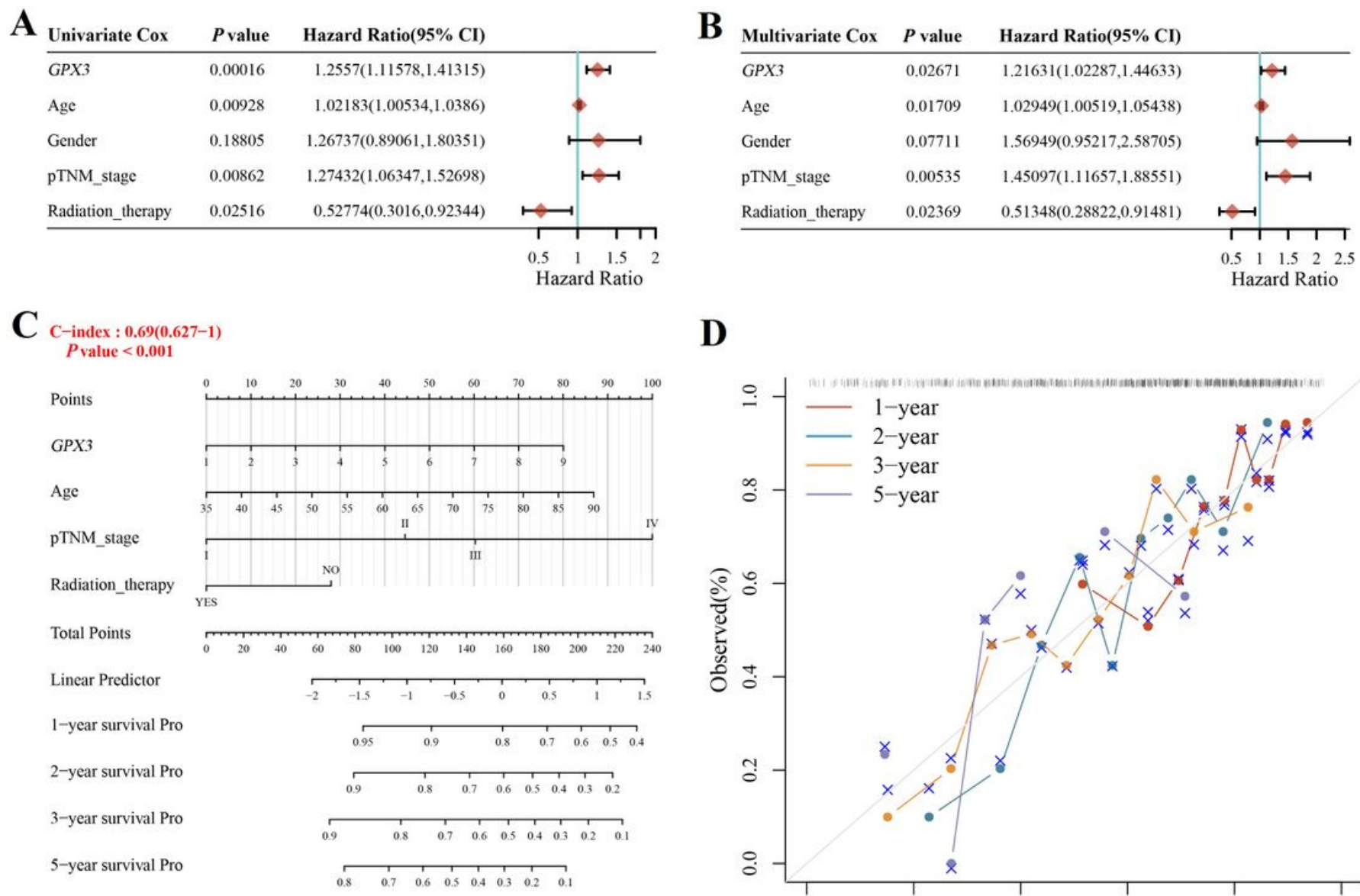

\section{D}

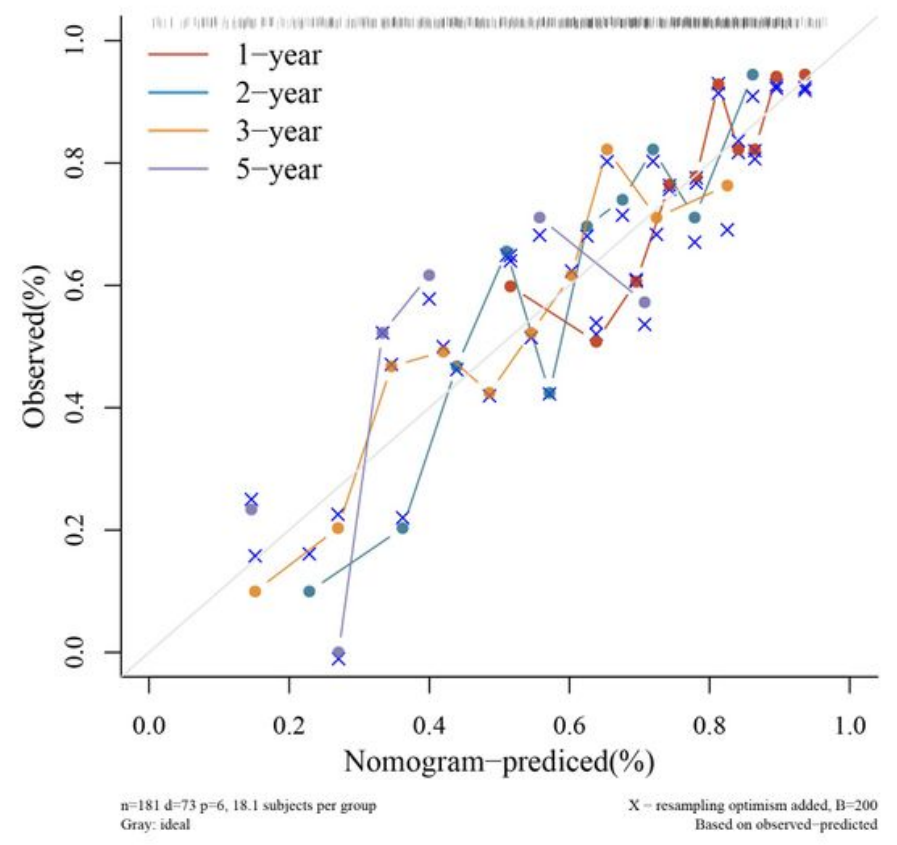

\section{Figure 10}

The nomogram predicted the probability survival in patients with STAD (A, B) Univariate and multivariate Cox proportional risk analyses of clinical parameters and risk scores in patients with STAD in the TCGA training cohort and CGGA validation cohort (C) Prognostic nomogram including age, pTNM stage and radiation therapy assessed probability survival of 1-, 2-,3-and 5-years (D) The calibration curves of the nomogram predicted survival in patients with STAD. 